\title{
Intracorporeal bi-directional pouch jejunojejunostomy following Roux-en-Y anastomosis: a simple reconstruction technique using an endoscopic linear stapler
}

\author{
Amy Kim, Chang Seok Ko, Byung Sik Kim, Hee Sung Kim \\ Department of Surgery, University of Ulsan College of Medicine, Asan Medical Center, Seoul, Republic of Korea
}

Videosurgery Miniinv 2021; 16 (4): 704-709

DOI: https://doi.org/10.5114/wiitm.2021.105720

\begin{abstract}
Introduction: Intracorporeal pouch jejunojejunostomy (JJ) is a technically challenging procedure during totally laparoscopic gastrectomy (TLG).

Aim: This study introduced a new method involving the construction of an intracorporeal bi-directional pouch J using an endoscopic linear stapler and analyzed the surgical outcomes of this method, including JJ complications.

Material and methods: We retrospectively reviewed the medical records of 168 patients who underwent laparoscopic gastrectomy with intracorporeal bi-directional pouch JJ between November 2017 and October 2018 at Asan Medical Center.

Results: The construction of an intracorporeal bi-directional pouch JJ took an average of $10 \mathrm{~min}$. No postoperative bleeding or anastomotic strictures related to JJ reconstruction occurred after this procedure. No mortality occurred during follow-up.

Conclusions: Intracorporeal bi-directional pouch JJ using an endoscopic linear stapler is a safe and simple procedure. It is a feasible option to reduce JJ stricture after TLG in patients with gastric cancer.
\end{abstract}

Key words: laparoscopy, complication, gastrectomy, stomach neoplasm, anastomosis.

\section{Introduction}

First introduced in 1991, laparoscopic gastrectomy is now considered a standard treatment for early gastric cancer based on a growing body of evidence demonstrating its technical feasibility and safety [1-5]. As the oncologic and surgical outcomes of laparoscopic gastrectomy have reached a certain level, much attention has been focused on the quality of life and nutritional outcomes of patients with gastric cancer after surgery as well as minimally invasive approaches such as intracorporeal anastomosis [6-9]. To improve patient quality of life and nutritional status after gastrectomy, ab-oral pouch jejunojejunos- tomy (JJ) was introduced. Specifically, ab-oral pouch J was developed to increase the preservative and digestive capacity of food materials and reduce bile reflux $[10,11]$.

Several recent studies have assessed patient quality of life and nutritional outcomes in relation to the construction of jejunal ab-oral pouches. Gertler et al. performed a meta-analysis of randomized controlled trials (RCTs) on pouch reconstruction after total gastrectomy (TG) for patients with gastric cancer published between 1987 and 2008 [12]. The authors concluded that pouch construction did not increase morbidity, mortality, or operative time, but it significantly increased patient quality of life. An RCT in

\section{Address for correspondence}

Hee Sung Kim, Department of Surgery, University of Ulsan College of Medicine, Asan Medical Center, Seoul, Republic of Korea,

e-mail: os-khs@hanmail.net 
2000 investigated the benefits of the ab-oral pouch, reporting its advantages in lipid absorption and improving quality of life among patients who received TG [11]. However, other studies have reported no significant advantage of the ab-oral pouch in improving patient quality of life after TG $[13,14]$. Thus, there is no consensus regarding the effectiveness of $a b$ oral pouch construction. Furthermore, none of the studies utilized minimally invasive approaches such as intracorporeal anastomosis. We hypothesized that the benefit of jejunal ab-oral pouch construction would become more evident after intracorporeal reconstruction is performed during laparoscopic gastrectomy.

\section{Aim}

Here, we introduce a new method: intracorporeal bi-directional pouch J using an endoscopic linear stapler. This study describes the surgical method for this novel technique and analyzes the surgical out comes including JJ-related complications.

\section{Material and methods}

\section{Patients}

Between November 2017 and October 2018, 168 patients with gastric cancer underwent laparoscopic gastrectomy with intracorporeal bi-directional pouch $J$ at Asan Medical Center, including 12 cases of robot gastrectomy. The operations were performed by 2 experienced laparoscopic gastric surgeons. This study was approved by the institutional review board of Asan Medical Center (approval number: 2018-1021).

\section{Clinical evaluation of surgical outcomes}

We reviewed medical records of patients to evaluate the clinical characteristics and surgical outcomes, including sex, age at operation, body mass index (BMI), history of previous abdominal surgery, American Society of Anesthesiologists (ASA) score, tumor size, tumor-node-metastasis (TNM) stage according to the American Joint Committee on Cancer Staging Manual $7^{\text {th }}$ edition, number of harvested lymph nodes, number of metastatic lymph nodes, proximal and distal resection margins, time for operation, number of cases converted to open laparotomy, date of the first passage of flatus, and length of hospital stay [15]. Length of hospital stay was estimated postoperatively; when a patient was readmitted within
30 days of surgery, the length of readmission stay was also considered. Postoperative mortality was defined as mortality within 30 days of surgery.

We defined early complications as surgery-related complications occurring within 30 days of surgery, while late complications were defined as those occurring > 30 days postoperatively. JJ-related complications were defined as events located in and around the J, including extraluminal bleeding, intraluminal bleeding, anastomosis leakage, and anastomosis stricture. These were diagnosed based on computed tomography, upper gastrointestinal series, and esophagogastroduodenoscopy findings or clinical signs and symptoms. The severity of complications was classified according to the Clavien-Dindo classification system [16].

\section{Surgical technique}

The procedure was performed with the patients placed in the reverse Trendelenburg position, with 5 ports. The first $12-\mathrm{mm}$ port was placed under the umbilicus using an open method; through this port, pneumoperitoneum was created. Under laparoscopic visualization, 12- and 5-mm trocars were inserted on both sides of the upper abdomen. Liver retraction was performed using the triangle method [17].

After the esophagojejunostomy (EJ) or gastrojejunostomy (GJ) reconstruction, a side-to-side jejunostomy was created by making entry holes in the efferent loop of the jejunum 40-50 cm from the EJ/ GJ and in the afferent loop of the jejunum 6-8 cm from the stump (Photo $1 \mathrm{~A}$ ). After construction of the JJ with a linear endostapler (ECHELON FLEX 60, blue cartridge; Photo $1 \mathrm{~B}$ ), instead of closing the common entry hole, anchoring sutures using black silk 3-0 were placed in the 6-o'clock position of the common hole (Photo $1 \mathrm{C}$ ) and 6-8 cm distal from the common hole (Photo 1 D). The JJ was then flipped anti-clockwise using the tagging sutures and another endostapler was inserted through the common hole (Photo $1 \mathrm{E}$ ). In this way, the anastomosis was extended to $12 \mathrm{~cm}$ in length (Photo $1 \mathrm{~F}$ ). After closure of the common opening, the "pouch" was positioned in the left upper quadrant (LUQ) area and the intermesenteric and Petersen's spaces were closed with black silk 3-0 sutures (Photo $1 \mathrm{G}$ ).

The difference between conventional side-toside anti-peristaltic $\mathrm{J}$ and the novel procedure described in this study is the bi-directional reconstruc- 

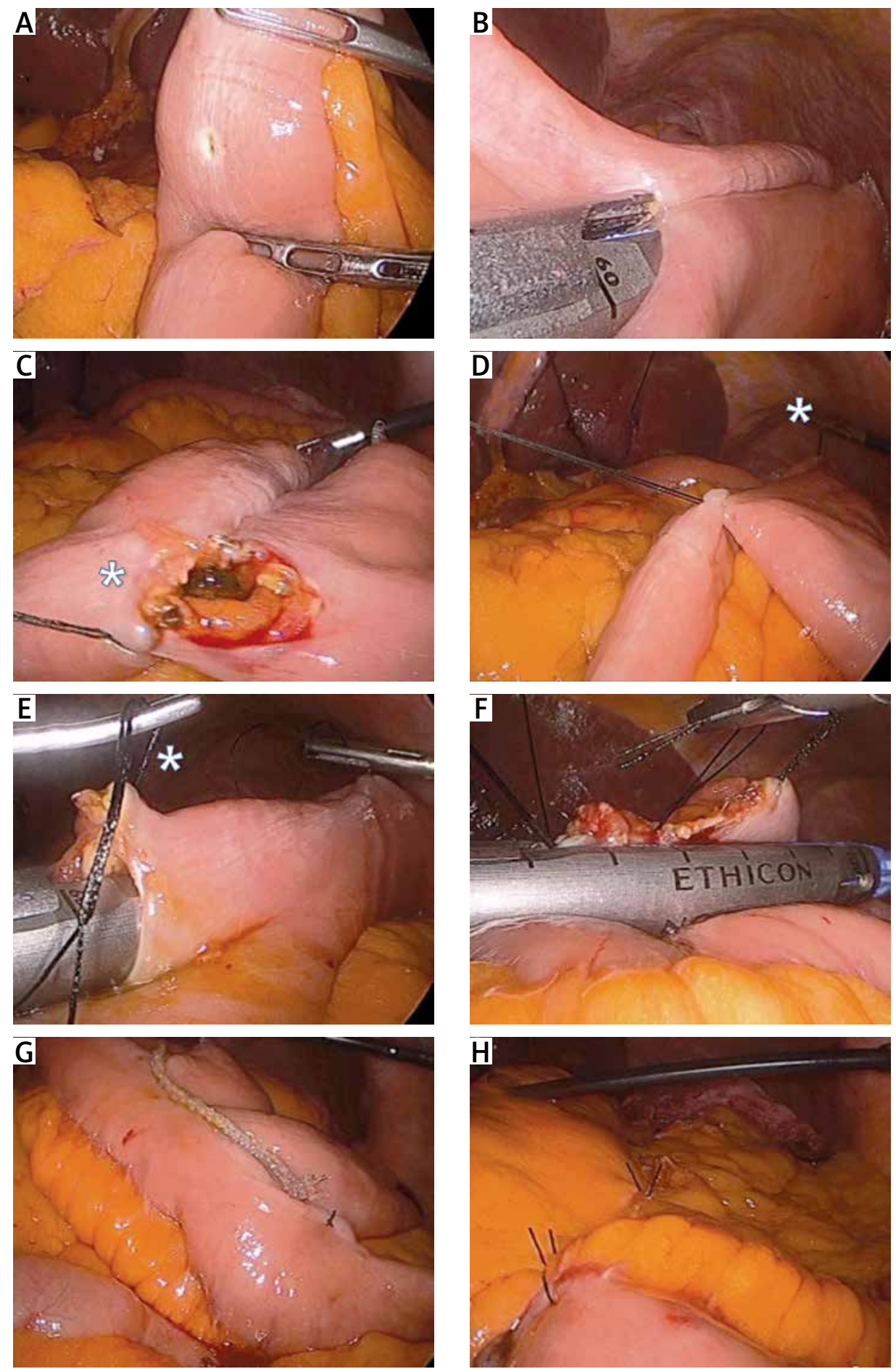

Photo 1. Image captures of the procedure to perform intracorporeal bi-directional pouch jejunojejunostomy. A - After enterotomies are made, B - a side-to-side J is made using a 60-mm endoscopic linear stapler. The asterisks in (C), (D), and (E) indicate tagging sutures made on the 6-o'clock side of the common hole. C - Another suture is made $6-8 \mathrm{~cm}$ from the first tagging suture. D - Using these tagging sutures, the JJ is rotated clockwise so that the endoscopic linear stapler can enter the common hole. $\mathbf{E}-$ The common hole is closed using an endoscopic stapler. F - The constructed JJ with an ab-oral pouch. G - The intermesenteric spaces are closed with interrupted sutures 
tion using two 60-mm endoscopic linear staplers. Second, the passage disturbance of the efferent loop was minimized by avoiding stapling of the common entry hole between the efferent and afferent loops.

\section{Results}

\section{Patient characteristics}

This study included 107 male and 61 female patients. The median age at surgery was 62 years, and the median BMI was $23.6 \mathrm{~kg} / \mathrm{m}^{2}$. Forty-two (25\%) patients had a history of previous abdominal surgery. All operations were performed totally intracorporeally by laparoscopy and no cases were convert-

Table I. Demographic characteristics of patients who underwent TLG with bi-directional pouch JJ

\begin{tabular}{|c|c|}
\hline Variables & Value \\
\hline Age [years] median & $62(35-84)$ \\
\hline \multicolumn{2}{|l|}{ Sex, $n(\%):$} \\
\hline Male/female & $\begin{array}{c}107(63.7) / \\
61(36.3)\end{array}$ \\
\hline BMI $\left[\mathrm{kg} / \mathrm{m}^{2}\right]$ median & $23.6(15.8-34.4)$ \\
\hline Previous abdominal surgery history, $n(\%)$ & $42(25.0)$ \\
\hline \multicolumn{2}{|l|}{ ASA score, $n(\%)$ : } \\
\hline 1 & $59(35.1)$ \\
\hline 2 & $102(60.7)$ \\
\hline 3 & $7(4.2)$ \\
\hline Follow-up period [months] median & $10(3-16)$ \\
\hline Tumor size $[\mathrm{cm}]$ mean \pm SD & $3.6 \pm 2.4$ \\
\hline $\begin{array}{l}\text { Proximal resection margin }[\mathrm{cm}] \\
\text { mean } \pm \mathrm{SD}\end{array}$ & $2.6 \pm 3.0$ \\
\hline Distal resection margin $[\mathrm{cm}]$ mean $\pm \mathrm{SD}$ & $11.1 \pm 4.5$ \\
\hline $\begin{array}{l}\text { Number of harvested lymph nodes } \\
\text { (mean } \pm \text { SD) }\end{array}$ & $29.5 \pm 12.4$ \\
\hline $\begin{array}{l}\text { Number of positive lymph nodes } \\
\text { (mean } \pm \text { SD) }\end{array}$ & $0.9 \pm 3.0$ \\
\hline \multicolumn{2}{|l|}{ TNM stage, $n(\%)$ : } \\
\hline la & $102(60.7)$ \\
\hline $\mathrm{Ib}$ & $18(10.7)$ \\
\hline Ila & $18(10.7)$ \\
\hline $\mathrm{Ilb}$ & $14(8.3)$ \\
\hline IIIa & $9(5.4)$ \\
\hline$I I I b$ & $6(3.6)$ \\
\hline IIIC & $1(0.6)$ \\
\hline
\end{tabular}

$T L G$ - totally laparoscopic gastrectomy, BMI - body mass index, ASA - American Society of Anesthesiologists, BMI - body mass index. ed to an open laparotomy. An ASA score of 2 was the most common in the preoperative examination, followed by a score of 1 and 3 .

\section{Pathological outcomes}

The mean tumor size was $3.6 \mathrm{~cm}$, with a mean proximal resection margin of $2.6 \mathrm{~cm}$ and a mean distal margin of $11.1 \mathrm{~cm}$. An average of 31.9 lymph nodes were harvested and 0.9 lymph nodes were positive for metastatic cells. There were $102(60.7 \%)$, 18 (10.7\%), 18 (10.7\%), 14 (8.3\%), 9 (5.4\%), 6 (3.6\%), and $1(0.6 \%)$ patients with TNM stages IA, IB, IIB, IIB, IIIA, IIIB, and IIIC, respectively (Table I).

\section{Early surgical outcomes}

For most patients, liquid and soft diets were initiated on the first and second days after surgery, respectively, regardless of the passage of flatus. The mean operative time was $172.1 \pm 47.5 \mathrm{~min}$, and JJ pouch formation time was $10.0 \pm 2.5 \mathrm{~min}$. No case required conversion to open laparotomy. On average, the first flatus passed within 3.5 days of surgery, and the patients were discharged at 7.0 days postoperatively (Table II).

Table III shows the postoperative complications in patients who underwent TLG with bi-directional pouch JJ. Luminal bleeding and extraluminal bleeding occurred in 3 patients after surgery. Esophagojejunostomy leakage occurred in 2 patients and duodenal stump leakage occurred in 1 patient during follow-up. Three patients experienced gastrojeju-

Table II. Early surgical outcomes of patients who underwent TLG with bi-directional pouch JJ $(n=168)$

\begin{tabular}{|c|c|}
\hline Variables & Value \\
\hline \multicolumn{2}{|l|}{ Type of operation, $n(\%)$ : } \\
\hline TLDG/TLTG & $77(45.8) / 91(54.2)$ \\
\hline Total operation time $[\mathrm{min}]$ mean \pm SD & $172.1 \pm 47.5$ \\
\hline $\begin{array}{l}\text { Time for JJ pouch formation [min] } \\
\text { mean } \pm \text { SD }\end{array}$ & $10.0 \pm 2.5$ \\
\hline First passage of flatus [day] mean \pm SD & $3.5 \pm 0.9$ \\
\hline Painkiller administration (median) & $2(0-48)$ \\
\hline $\begin{array}{l}\text { Length of hospital days [day] } \\
\text { mean } \pm \text { SD }\end{array}$ & $7.0 \pm 4.5$ \\
\hline
\end{tabular}

$T L G$ - totally laparoscopic gastrectomy, TLDG - totally laparoscopic distal gastrectomy, TLTG - totally laparoscopic total gastrectomy, JJ - jejunojejunostomy, SD - standard deviation. 
Table III. Surgical complications and mortality of patients who underwent TLG with bi-directional pouch JJ

\begin{tabular}{|c|c|c|}
\hline Variables & TLDG & TLTG \\
\hline \multicolumn{3}{|l|}{ Complications: } \\
\hline Anastomotic leakage & 0 & 0 \\
\hline EJ leakage & 0 & 2 \\
\hline J leakage & 0 & 0 \\
\hline Duodenal stump leakage & 0 & 1 \\
\hline Anastomotic stricture & 0 & 0 \\
\hline EJ stricture & 3 & 0 \\
\hline J stricture & 0 & 0 \\
\hline Pancreatic fistula & 0 & 1 \\
\hline Luminal bleeding & 1 & 1 \\
\hline Extraluminal bleeding & 1 & 0 \\
\hline Ileus & 2 & 6 \\
\hline Intra-abdominal fluid collection & 2 & 1 \\
\hline Wound problem & 4 & 2 \\
\hline Others & 2 & 4 \\
\hline Medical complications & 1 & 0 \\
\hline Mortality & 0 & 0 \\
\hline
\end{tabular}

nostomy stricture, while no patients experienced J) stricture. Pancreatic fistula, wound infection, intra-abdominal fluid collection, and ileus were noted in $1,5,8$, and 5 patients, respectively. Five patients experienced medical complications, including postoperative pneumonia, asthma attack, and enteritis. No mortality occurred during follow-up.

\section{Discussion}

Laparoscopic gastrectomy with intracorporeal reconstruction is a technique that requires well-trained skills and sufficient experience. Likewise, intracorporeal pouch $J \mathrm{~J}$ is a technically challenging procedure even for highly experienced gastric cancer surgeons. This study, however, introduced the method of intracorporeal bi-directional pouch JJ using an endoscopic linear stapler, which grafts the JJ pouch onto a laparoscopic gastrectomy. It was designed to more simply and easily perform intracorporeal anastomosis.

Although various complications have been reported after laparoscopic gastrectomy, strictures at the JJ site have rarely been mentioned. JJ stricture is usually caused by adhesion and could be aggravated by postoperative fluid collection, bleeding, trocar site, and staple line. Adhesion at this site may lead to JJ kinking, twisting, or stricture. Although the incidence of JJ stricture after laparoscopic Roux-en-Y bypass is low, around $0.5-1.2 \%$, all patients with this complication require laparoscopic or open surgery [18-20]. In other words, JJ stricture is a rare but potentially fatal complication that almost always requires intervention or reoperation.

In this study, we demonstrated favorable surgical outcomes, including JJ-related complications. Specifically, no JJ strictures were reported during the follow-up period. We hypothesized that this was because of the capacity of the pouch; even when adhesion or kinking developed, there was enough space for food passage, thus preventing stricture. Unlike conventional J, which induces stricture between the Roux and afferent limb during the process of closing the common channel of JJ, this novel method decreases the risk of efferent loop narrowing. In summary, we believe that the intracorporeal bi-directional pouch JJ could be an effective procedure that can reduce the occurrence of JJ strictures.

The mean total operative time was $172.1 \mathrm{~min}$, with an average of 10 min required for pouch construction. However, as no data were available on the time required for the construction of conventional $\mathrm{JJ}$, direct comparison of these times was not possible; however, our results indicate that the pouch formation is feasible. Further, although this was not a comparative study, our method showed favorable outcomes and safety. Our method showed favorable surgical outcomes, with a shorter length of hospital stay and a lower morbidity rate than those reported in previous studies [13, 14].

Our study has several limitations. First, it was a retrospective single-center study. In addition, JJ stricture is a rare complication; therefore, the number of patients included in this study was not large enough to properly evaluate the incidence of complications. Additionally, this procedure was developed $<1$ year ago; thus, the primary goal of this study was to describe the procedure and our early experiences. However, our results are consistent with those reported by Gunabushanam et al. regarding the development of JJ strictures within 1 month of surgery [21]. Considering the early occurrence of JJ stricture, we expect our findings to reflect valid results de- 
spite the relatively short follow-up period. Another limitation was the descriptive and cross-sectional nature of this study. Therefore, a comparative study between patients receiving ab-oral pouch and conventional J may provide additional insights on this procedure. Further studies with more patients and continuous follow-up are needed. Further, additional research on the nutritional gains and quality of life could be beneficial for patients.

\section{Conclusions}

The current study results show that intracorporeal bi-directional pouch JJ using an endoscopic linear stapler is a safe and simple procedure. It is a feasible alternative to reduce JJ stricture after TLG for patients with gastric cancer.

\section{Acknowledgments}

Amy Kim and Chang Seok Ko contributed equally as first author to this article.

\section{Conflict of interest}

The authors declare no conflict of interest.

\section{References}

1. Kim HH, Hyung WJ, Cho GS, et al. Morbidity and mortality of laparoscopic gastrectomy versus open gastrectomy for gastric cancer: an interim report-a phase III multicenter, prospective, randomized trial (KLASS Trial). Ann Surg 2010; 251: 417-20.

2. Kim W, Kim HH, Han SU, et al. Decreased morbidity of laparoscopic distal gastrectomy compared with open distal gastrectomy for stage I gastric cancer: short-term outcomes from a multicenter randomized controlled trial (KLASS-01). Ann Surg 2016; 263: 28-35.

3. Kitano S, Iso Y, Moriyama M, et al. Laparoscopy-assisted Billroth I gastrectomy. Surg Laparosc Endosc 1994; 4: 146-8.

4. Kitano S, Shiraishi N, Fujii K, et al. A randomized controlled trial comparing open vs laparoscopy-assisted distal gastrectomy for the treatment of early gastric cancer: an interim report. Surgery 2002; 131 (1 Suppl): S306-11.

5. Zheng CY, Dong ZY, Zheng LZ, et al. Laparoscopic D2 plus complete mesogastrium excision using the "enjoyable space" approach versus conventional D2 total gastrectomy for local advanced gastric cancer: short-term outcomes. Videosurgery Miniinv 2020; 15: 58-69.

6. Kim HH, Han SU, Kim MC, et al. Effect of laparoscopic distal gastrectomy vs open distal gastrectomy on long-term survival among patients with stage I gastric cancer: the KLASS-01 randomized clinical trial. JAMA Oncol 2019; 5: 506-13.

7. Kim YW, Baik YH, Yun YH, et al. Improved quality of life outcomes after laparoscopy-assisted distal gastrectomy for early gastric cancer: results of a prospective randomized clinical trial. Ann Surg 2008; 248: 721-7.

8. Kobayashi D, Kodera Y, Fujiwara M, et al. Assessment of quality of life after gastrectomy using EORTC QLQ-C30 and STO22. World J Surg 2011; 35: 357-64.

9. Yu J, Huang C, Sun Y, et al. Effect of laparoscopic vs open distal gastrectomy on 3-year disease-free survival in patients with locally advanced gastric cancer: the CLASS-01 randomized clinical trial. JAMA 2019; 321: 1983-92.

10. Kalmár K, Cseke L, Zámbó K, et al. Comparison of quality of life and nutritional parameters after total gastrectomy and a new type of pouch construction with simple Roux-en-Y reconstruction: preliminary results of a prospective, randomized, controlled study. Dig Dis Sci 2001; 46: 1791-6.

11. Horváth OP, Kalmár K, Cseke L, et al. Nutritional and life-quality consequences of aboral pouch construction after total gastrectomy: a randomized, controlled study. Eur J Surg Oncol 2001; 27: 558-63.

12. Gertler R, Rosenberg R, Feith M, et al. Pouch vs. no pouch following total gastrectomy: meta-analysis and systematic review. Am J Gastroenterol 2009; 104: 2838-51.

13. Ito Y, Yoshikawa T, Fujiwara M, et al. Quality of life and nutritional consequences after aboral pouch reconstruction following total gastrectomy for gastric cancer: randomized controlled trial CCG1101. Gastric Cancer 2016; 19: 977-85.

14. Tanaka C, Kanda M, Murotani K, et al. Long-term quality of life and nutrition status of the aboral pouch reconstruction after total gastrectomy for gastric cancer: a prospective multicenter observational study (CCOG1505). Gastric Cancer 2019; 22: 607-16.

15. The Union for International Cancer Control's. TNM [Internet]. Geneva, Switzerland: The Union for International Cancer Control's; 2020 [updated 2020 Sep 11; cited 2020 Nov 2]. Available from: https://www.uicc.org/resources/tnm.

16. Dindo D, Demartines N, Clavien PA. Classification of surgical complications: a new proposal with evaluation in a cohort of 6336 patients and results of a survey. Ann Surg 2004; 240: 205-13.

17. Lee IS, Kim TH, Yook JH, et al. A triangle method: simple suture retraction for the left lobe of the liver during laparoscopic gastric surgery. J Laparoendosc Adv Surg Tech A 2012; 22: 989-91.

18. Husain S, Ahmed AR, Johnson J, et al. Small-bowel obstruction after laparoscopic Roux-en-Y gastric bypass: etiology, diagnosis, and management. Arch Surg 2007; 142: 988-93.

19. Kamada T, Ohdaira H, Hoshimoto S, et al. Fluoroscopic balloon dilation for early jejunojejunostomy obstruction after gastrectomy with roux-en-Y reconstruction: a case series of three patients. Surg Case Rep 2020; 6: 108.

20. Lewis CE, Jensen C, Tejirian T, et al. Early jejunojejunostomy obstruction after laparoscopic gastric bypass: case series and treatment algorithm. Surg Obes Relat Dis 2009; 5: 203-7.

21. Gunabushanam G, Shankar S, Czerniach DR, et al. Small-bowel obstruction after laparoscopic Roux-en-Y gastric bypass surgery. J Comput Assist Tomogr 2009; 33: 369-75.

Received: 20.12.2020, accepted: 3.02.2021. 